\title{
Response to antiplatelet therapy in patients undergoing invasive treatment due to acute coronary syndrome complicated by cardiogenic shock
}

\author{
Wiktor Kuliczkowski ${ }^{1}$, Magdalena Cielecka-Prynda ${ }^{1}$, Bożena Karolko ${ }^{1}$, Konrad Kaaz ${ }^{1}$, Barbara Adamik ${ }^{2}$, \\ Dawid Bednarczyk ${ }^{1}$, Małgorzata Kobusiak-Prokopowicz ${ }^{1}$, Andrzej Mysiak ${ }^{1}$ \\ ${ }^{1}$ Department and Clinic of Cardiology, Wroclaw Medical University, Wroclaw, Poland \\ ${ }^{2}$ Department of Anesthesiology and Intensive Therapy, Wroclaw Medical University, Wroclaw, Poland
}

Adv Interv Cardiol 2020; 16, 4 (62): 418-421

DOI: https://doi.org/10.5114/aic.2020.101766

\begin{abstract}
A bstract
Introduction: There are limited data on platelet reactivity and response to antiplatelet drugs in patients with cardiogenic shock.

Aim: To assess platelet reactivity on dual antiplatelet therapy with acetylsalicylic acid (ASA) and ticagrelor, a novel potent P2Y12 receptor inhibitor, in patients with cardiogenic shock in the course of acute coronary syndrome (ACS) who received invasive treatment.

Material and methods: We enrolled 12 consecutive patients with ACS complicated by cardiogenic shock. To assess response to antiplatelet therapy during cardiogenic shock, only patients with symptoms persisting for at least 3 days and who completed a 5-day follow-up were included in the study. Patients received a loading dose of ASA (300 mg) and ticagrelor (180 mg), followed by a maintenance dose (ASA, $1 \times 75 \mathrm{mg}$; ticagrelor, $2 \times 90 \mathrm{mg}$ ). Blood samples for platelet function tests were collected. Platelet aggregation was assessed with a Multiplate whole-blood impedance aggregometer. Arachidonic acid (AA), adenosine diphosphate (ADP), and thrombin receptor-activating peptide (TRAP) were used as aggregation agonists.

Results: Response to antiplatelet therapy assessed by aggregometry showed numerically higher on-ASA platelet reactivity on day one and statistically significant higher on-ticagrelor platelet reactivity on day one in comparison with following days. There were 2 patients with high on ASA platelet reactivity and 3 with high on ticagrelor platelet reactivity, but only on the day one.

Conclusions: Some patients with cardiogenic shock in the course of ACS treated invasively show a lower response to ASA and ticagrelor only on the first day after invasive treatment, with a good response on subsequent days.
\end{abstract}

Key words: antiplatelet therapy, cardiogenic shock, myocardial infarction, ticagrelor.

S u m m a ry

There are limited data on platelet reactivity and response to antiplatelet drugs in patients with cardiogenic shock. In some patients, inadequate response to antiplatelet therapy is observed, which is additionally associated with a 2-fold higher risk of adverse events. As the incidence of cardiogenic shock is relatively low (about 5-6\%) and patients with shock are in a severe clinical condition, data on platelet reactivity and response to antiplatelet therapy in this population are limited.

\section{Introduction}

Antiplatelet therapy is one of the cornerstones of contemporary cardiology. In comparison with clopidogrel, novel potent antiplatelet drugs such as ticagrelor and prasugrel improve the prognosis of patients with acute coronary syndromes (ACSs) [1, 2]. On the other hand, ACS complicated by cardiogenic shock is still associated with a mortality rate of nearly $50 \%$ [3]. In some patients, inadequate response to antiplatelet therapy is observed, which is additionally associated with a 2-fold higher risk of adverse events [4]. As the incidence of cardiogenic shock is relatively low (about 5-6\%) and patients with shock are in a severe clinical condition, data on platelet reactivity and response to antiplatelet therapy in this population are limited.

\section{Corresponding author:}

Wiktor Kuliczkowski MD, PhD, Department and Clinic of Cardiology, Wroclaw Medical University, 213 Borowska St, 50-556 Wroclaw, Poland, phone: +48 603707 093, e-mail: wiktor.kuliczkowski@umed.wroc.pl

Received: 2.06.2020, accepted: 22.08.2020. 


\section{Aim}

The aim of this study was to assess platelet reactivity on dual antiplatelet therapy with acetylsalicylic acid (ASA) and ticagrelor, a novel potent $\mathrm{P} 2 \mathrm{Y}_{12}$ receptor inhibitor, in patients with cardiogenic shock in the course of ACS who received invasive treatment.

\section{Material and methods}

We enrolled consecutive patients with ACS complicated by cardiogenic shock. To assess the response to antiplatelet therapy during cardiogenic shock, only patients with symptoms persisting for at least 3 days were included, while only patients who completed a 5-day follow-up were included in further analysis. Cardiogenic shock was defined as hypotension (systolic blood pressure $<90 \mathrm{~mm} \mathrm{Hg}$ ) despite adequate left ventricular filling pressure, with concomitant signs and symptoms of end-organ hypoperfusion. All participants were treated according to the current guidelines for the management of patients with ACS $[5,6]$. All patients underwent coronary angiography and angioplasty of the infarct-related artery. Before referral to a hemodynamics laboratory, they received a loading dose of ASA (300 mg) and ticagrelor $(180 \mathrm{mg})$. Blood samples for platelet function tests were first obtained within $24 \mathrm{~h}$ after coronary angioplasty, and then on subsequent days in the morning. Three patients who initially met the inclusion criteria, but in whom blood samples were obtained only for 2 subsequent assays, were excluded from the study.

\section{Platelet aggregation}

Platelet aggregation was assessed with a Multiplate whole-blood impedance aggregometer (Roche, Basel, Switzerland). Blood was collected into test tubes containing hirudin $(25 \mu \mathrm{g} / \mathrm{ml})$ as an anticoagulant (Sarstedt, Germany). The aggregation agonists used were arachidonic acid (AA) at a target concentration of $0.5 \mathrm{mM}$, adenosine diphosphate (ADP) at a target concentration of $6.4 \mu \mathrm{M}$, and thrombin receptor-activating peptide (TRAP) at a target concentration of $32 \mu \mathrm{M}$. The reagents were provided by Roche. Platelet aggregation was assessed within $2 \mathrm{~h}$ from blood sampling and was presented as the area under the curve of aggregation measured in arbitrary units (AU). Each aggregation measurement was performed in duplicate, and the mean value was calculated. In the case of a $10 \%$ difference between measurements, the result was rejected and the measurement was repeated. Arachidonic acid-induced aggregation was used as a measure of response to ASA with a cutoff $A U$ value below 30; ADP-induced aggregation was used as a measure of response to ticagrelor with a cutoff $A U$ value below 48; and TRAP-induced aggregation was used as a measure of baseline platelet reactivity independent from ASA and ticagrelor.

\section{Statistical analysis}

Statistical analysis was conducted using the Statistica 9.0 software (StatSoft, Tulsa, Oklahoma, United States). For all patients, mean values and standard deviations were calculated, and the normality of the distribution of the parameters was tested with the Kolmogorov-Smirnov, Lilliefors, and Shapiro-Wilk tests. Differences in platelet reactivity between days 1 to 5 and a trend analysis was measured using the Friedman analysis of variance.

\section{Results}

The study included 12 patients with cardiogenic shock in the course of myocardial infarction. The clinical characteristics of patients are presented in Table I. On enrollment, all patients met the criteria for the diagno-

Table I. Clinical characteristics of the study group $(n=12)$

\begin{tabular}{|c|c|}
\hline Parameter & Value \\
\hline Age, mean (SD) [years] & $77(9)$ \\
\hline Sex, female/male, $n$ & $2 / 10$ \\
\hline \multicolumn{2}{|l|}{ Infarct-related artery, $n$ : } \\
\hline$\underline{L M C A}$ & 3 \\
\hline$\underline{L A D}$ & 8 \\
\hline$C x$ & 1 \\
\hline \multicolumn{2}{|l|}{ ACS, $n:$} \\
\hline STEMI & 3 \\
\hline NSTEMI & 9 \\
\hline Previous $\mathrm{PCl}, n$ & 7 \\
\hline Previous CABG, $n$ & 2 \\
\hline Diabetes, $n$ & 4 \\
\hline Hypertension, $n$ & 10 \\
\hline Hyperlipidemia, $n$ & 11 \\
\hline Renal failure, $n$ & 2 \\
\hline Hemoglobin, mean (SD) [g/dl] & $9.6(2.0)$ \\
\hline WBC, mean (SD) $\left[\times 10^{3} / \mathrm{mm}^{3}\right]$ & $15.1(6.6)$ \\
\hline Platelets, mean (SD) $\left[\times 10^{3} / \mathrm{mm}^{3}\right]$ & $216(88)$ \\
\hline eGFR, mean (SD) $[\mathrm{ml} / \mathrm{min} / \mathrm{kg}]$ & $59(39)$ \\
\hline Creatinine, mean (SD) [g/dl] & $1.5(0.7)$ \\
\hline LVEF, mean (SD) (\%) & $35(9)$ \\
\hline IABP, $n$ & 3 \\
\hline \multicolumn{2}{|l|}{ Catecholaminergic agents, $n$ : } \\
\hline Total & 12 \\
\hline Norepinephrine & 10 \\
\hline Dobutamine & 8 \\
\hline \multicolumn{2}{|l|}{ Bleeding by BARC, $n$ : } \\
\hline Type 2 & 4 \\
\hline Type 3a & 1 \\
\hline
\end{tabular}

ACS - acute coronary syndrome, BARC - Bleeding Academic Research Consortium, CABG - coronary artery bypass grafting, $C X$ - circumflex artery, eGFR estimated glomerular filtration rate, IABP-intra-aortic balloon pump, $L A D$ - left anterior descending artery, $L M C A$ - left main coronary artery, $L V E F$ - left ventricular ejection fraction, NSTEMI, non-ST-segment elevation myocardial infarction, $\mathrm{PCl}$ - percutaneous coronary intervention, STEMI - ST-segment elevation myocardial infarction, WBC - white blood cells. 
Table II. Platelet aggregation results

\begin{tabular}{lccccc} 
Agonist & \multicolumn{5}{c}{ Aggregation [AU] } \\
\cline { 2 - 6 } & Day 1 & Day 2 & Day 3 & Day 4 & Day 5 \\
\hline AA $^{\mathrm{a}}$, mean (SD) & $21.0(5.6)$ & $10.5(5.8)$ & $12.5(9.8)$ & $17.0(11.1)$ & $16.5(9.1)$ \\
\hline ADP $^{\mathrm{b}}$, mean (SD) & $44.0(35.3)^{*}$ & $16.5(3.5)$ & $15.0(11.3)$ & $15.0(2.8)$ & $13.5(10.6)$ \\
\hline TRAPc, mean (SD) $^{\text {TRA }}$ & $67.3(13.4)$ & $61.0(4.2)$ & $72.5(0.7)$ & $75.5(33.2)$ & $78.0(22.6)$
\end{tabular}

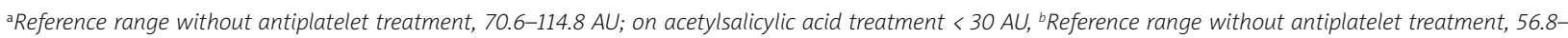
$113.0 \mathrm{AU}$; on P2Y12 antagonist treatment < $48 \mathrm{AU}$, 'Reference range, 83.6-128.0 AU. AA - arachidonic acid, ADP - adenosine diphosphate, AU - arbitrary units, TRAP - thrombin receptor-activating peptide. ${ }^{*} P<0.05$ for Day 1 in comparison to Day $2-5$.

sis of cardiogenic shock and were treated with catecholamine infusion. In 3 patients, it was possible to discontinue the use of catecholamines on day 3 of hospitalization, while the remaining patients were maintained on catecholamine treatment throughout the study. One patient

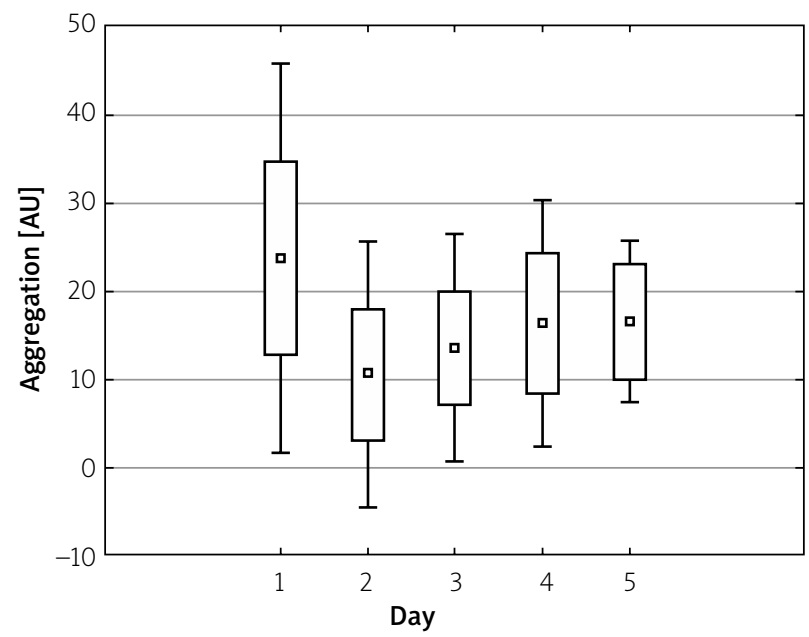

Figure 1. Response to acetylsalicylic acid assessed by arachidonic acid-induced aggregation (square - mean, box - standard error, whiskers - standard deviation)

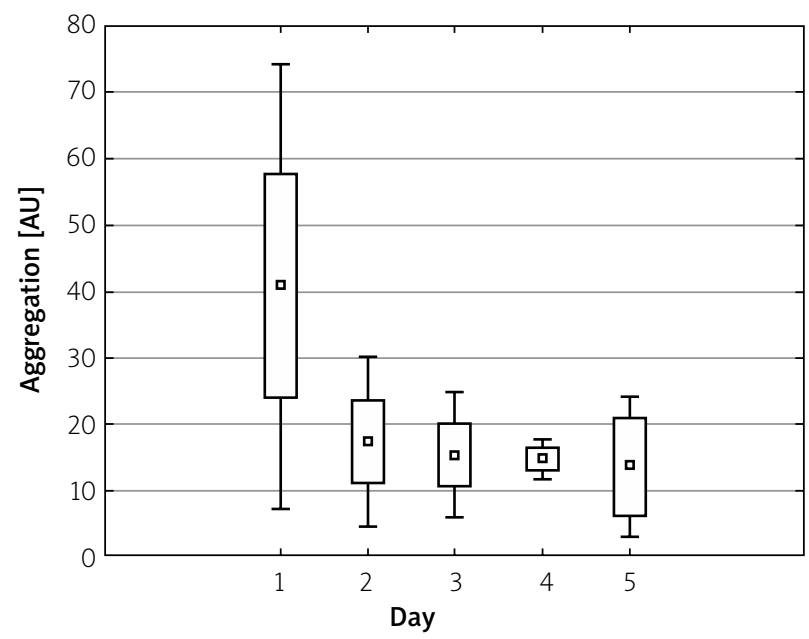

Figure 2. Response to ticagrelor assessed by adenosine diphosphate-induced aggregation (square - mean, box - standard error, whiskers - standard deviation) died on day 6 and another one on day 7 due to multiorgan failure. The other patients survived until discharge. During follow-up at the cardiac intensive care unit, we did not observe stent thrombosis, stroke, or recurrent myocardial infarction.

The first blood specimen for platelet aggregation was obtained after a mean time of $12 \mathrm{~h} \pm 65 \mathrm{~min}$ after a loading dose of antiplatelet agents.

Response to antiplatelet therapy assessed by aggregometry showed numerically higher on-ASA platelet reactivity on day one and statistically significant higher on-ticagrelor platelet reactivity on day one in comparison with following days. In the case of TRAP-induced aggregation, a non-significant tendency for higher on-treatment platelet reactivity was observed since day 2 of hospitalization (Table II, Figures 1-3).

In 2 patients, an inadequate response to ASA was observed on day 1 , and in 3 patients, to ticagrelor. On subsequent assays, all patients showed an adequate response to both antiplatelet drugs.

\section{Discussion}

Our results showed that some patients with ACS complicated by cardiogenic shock who receive invasive

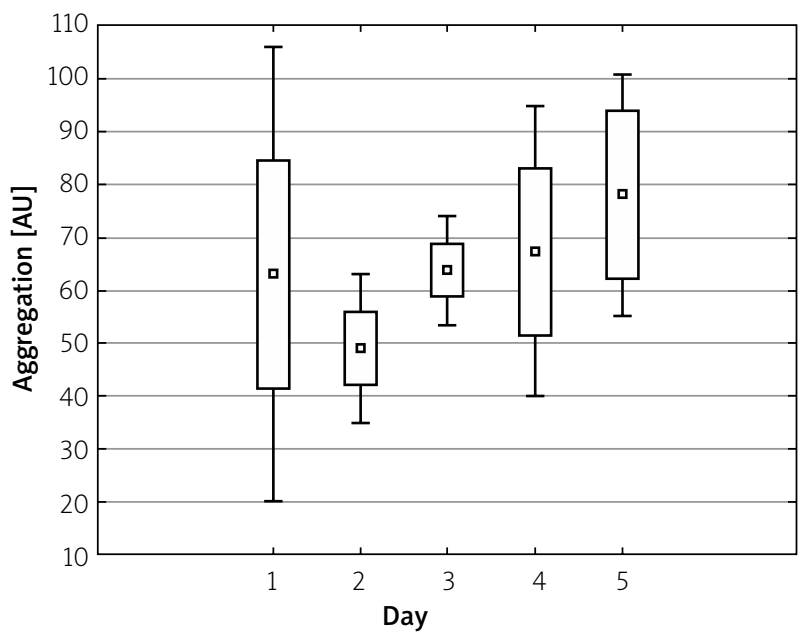

Figure 3. Baseline platelet reactivity assessed by platelet aggregation induced by thrombin receptor-activating peptide (square - mean, box - standard error, whiskers - standard deviation) 
treatment had an inadequate response to dual antiplatelet therapy on day 1 of hospitalization, with an adequate response both to ASA and ticagrelor on subsequent days. Orban et al. [7] reported that patients with ACS after successful resuscitation who underwent targeted temperature management showed a worse response to antiplatelet therapy on day 3 of hospitalization. However, the reason for this finding is unknown [7]. In our study, none of the patients underwent resuscitation or targeted temperature management. Therefore, a comparison between the studies is difficult. On the other hand, in a study by Tilemann et al. [8], inadequate response to antiplatelet therapy in patients with cardiogenic shock was associated with early stent thrombosis, with no effect on late stent thrombosis and repeat myocardial infarction. However, the authors assessed the response to treatment only on day 1 of hospitalization. In our study, we also observed an inadequate response in 5 patients, but only on day 1 . We did not observe stent thrombosis or repeat myocardial infarction.

A possible explanation of our results is the malabsorption of oral antiplatelet drugs from the stomach and duodenum in the state of shock, when there is hypoperfusion of the gastrointestinal tract aggravated by catecholamines. Notably, in our patients there was no use of morphine, which is known for its influence on the antiplatelet drug effect via gastrointestinal absorption interference [9].

Baseline platelet reactivity, assessed with TRAP-induced aggregation, showed an increasing tendency on subsequent assays. This may reflect a homeostatic mechanism whereby platelets are produced in the bone marrow in response to the inhibitory effects of antiplatelet drugs on 2 other platelet signaling pathways, which was reported in our previous study on patients with ACS [10].

The most important limitation of our study is the small and highly selected study group. This is because of a low incidence of cardiogenic shock in patients with ACS treated invasively. Moreover, in order to meet the inclusion criteria, the signs and symptoms of cardiogenic shock had to persist for at least 3 days, and blood samples had to be obtained for 5 consecutive days.

\section{Conclusions}

Some patients with cardiogenic shock in the course of ACS treated invasively show a lower response to ASA and ticagrelor only on the first day after invasive treatment, with a good response on subsequent days.

\section{Conflict of interest}

The authors declare no conflict of interest.

\section{References}

1. Wiviott SD, Braunwald E, McCabe $\mathrm{CH}$, et al. Prasugrel versus clopidogrel in patients with acute coronary syndromes. N Engl J Med 2007; 357: 2001-15.

2. Wallentin L, Becker RC, Budaj A, et al. Ticagrelor versus clopidogrel in patients with acute coronary syndromes. N Engl J Med 2009; 361: 1045-57.

3. Ponikowski P, Voors AA, Anker SD, et al. Guidelines for the diagnosis and treatment of acute and chronic heart failure. Kardiol Pol 2016; 74: 1037-147.

4. Gross L, Aradi D, Sibbing D. Platelet function testing in patients on antiplatelet medications. Semin Thromb Hemost 2016; 42: 306-20.

5. Ibanez B, James S, Agewall S, et al.; ESC Scientific Document Group. 2017 ESC Guidelines for the management of acute myocardial infarction in patients presenting with ST-segment elevation: the Task Force for the management of acute myocardial infarction in patients presenting with ST-segment elevation of the European Society of Cardiology (ESC). Eur Heart J 2018; 39: 119-77.

6. Roffi M, Patrono C, Collet JP, et al. 2015 ESC guidelines for the management of acute coronary syndromes in patients presenting without persistent ST-segment elevation. Kardiol Pol 2016; 73: 1207-94

7. Orban M, Mayer K, Morath T, et al. The impact of therapeutic hypothermia on on-treatment platelet reactivity and clinical outcome in cardiogenic shock patients undergoing primary $\mathrm{PCI}$ for acute myocardial infarction: results from the ISAR-SHOCK registry. Thromb Res 2015; 136: 87-93.

8. Tilemann L, Mohr SK, Preusch M, et al. Platelet function monitoring for stent thrombosis in critically III patients with an acute coronary syndrome. J Interv Cardiol 2018; 31: 277-83.

9. Kubica J, Adamski P, Ostrowska M, et al. Morphine delays and attenuates ticagrelor exposure and action in patients with myocardial infarction: the randomized, double-blind, placebo-controlled IMPRESSION trial. Eur Heart J 2016; 37: 245-52.

10. Kuliczkowski W, Szewczyk M, Kaczmarski J, et al. Thrombin generation and platelet reactivity at hospital discharge and 6-month outcome after the acute coronary syndrome in diabetic and nondiabetic patients. Cardiology 2014; 128: 25-33. 